\title{
Group Dynamics in Gameful Collaborative Innovation Processes
}

\author{
Sarah-Kristin Thiel ${ }^{1}$, Jeanette Falk Olesen ${ }^{2}$, Kim Halskov ${ }^{2}$, and Ida \\ Larsen-Ledet ${ }^{1}$ \\ 1 Department of Computer Science, Aarhus University, Denmark thiel@cs.au.dk \\ ida.11@cs.au.dk \\ 2 Centre for Digital Creativity, Aarhus University, Denmark jfo@cc.au.dk \\ halskov@cavi.au.dk
}

\begin{abstract}
Creativity is widely recognized as a valuable trait. The aim of this research is to explore the usefulness of incorporating elements of both game and place into creativity methods, so called design games or gameful ideation methods. The present paper presents findings from a study in which a Gameful Idea Generation (GIG) was conducted. GIG is a collaborative creativity method that draws on concepts from design games and hackathons. We focus on intra-group dynamics in order to gain insights on how gamefulness influences collaborative creative processes. We analyse performance in relation to the level and nature of collaboration displayed during group-based ideation. Our insights provide relevant pointers for the design of future collaborative creativity enhancing technologies.
\end{abstract}

Keywords: creativity methods $\cdot$ gamefulness $\cdot$ collaboration · ideation · design games $\cdot$ group dynamics $\cdot$ divergent thinking. ${ }^{3}$

\section{Introduction}

Over the last decade, research has introduced a plethora of creativity methods to support idea generation and facilitate innovation processes at large. With studies having suggested that gamefulness can promote creativity (e.g. [12]), concepts known from games have lately been introduced into creativity methods coining so called design games $[1,10]$. This paper investigates how collaboration unfolds in a creative design process and how a gameful framing affects the nature of the collaboration (i.e. intra-group dynamics) and the outcomes of the ideation process (i.e. quality of ideas).

We do so by drawing on data collected during a one day event with high school students in which we conducted a Gameful Idea Generation (introduced in [19]). Based on gathered insights on how participants worked together in the collaborative creative process, we hope to provide pointers that can be used to inform the design of technologies to support collaborative creativity. Our insights

3 The final authenticated version is available online at https://doi.org/10.1007/978-3030-28011-6_16. 
can furthermore contribute to research on hackathons, which are usually not designed to support learners [7], even when used in educational settings. Structuring hackathons via methods such as Gameful Idea Generations has potential in this perspective.

\section{Background}

This work focuses on collaborative ideation processes that take place in a setting where novel and meaningful solutions for a given problem are generated, prioritized and demonstrated (e.g. business meeting). With the term collaborative, we refer to a setting where two or more persons work together in a joint effort.

\subsection{Card-based Gameful Creativity methods}

The use of tangible cards is a well established approach to stimulate divergent thinking as well as guide design processes (e.g. $[9,15])$. During ideation, cards have been found to foster divergent thinking by triggering ideas that go beyond the present context allowing to creatively interpret possibilities [11].

For the context of this work, we are interested in those creativity methods that adopt principles known from games. Rationales for incorporating gamefulness into creative processes include that playing is said to stimulate creativity by facilitating the cognitive (i.e skill domain) and affective (i.e. eliciting positive emotions) dimensions of creative processes. Furthermore, being based on a set of rules, games follow systematic structures (e.g. sequences of interaction) that help generate a creative flow [13]. We posit that such a gameful (rule) structure might foster creativity $[13,22]$. Competition creates a tension that becomes a driving force [15], which can facilitate idea generation processes. Through balancing intrinsic pleasure (i.e. fun) and the level of difficulty (i.e. challenges), games achieve a state of flow that helps retain motivation throughout a game (or process) [4] and may also promote collaborative thinking $[8,14]$.

\subsection{Collaborative Innovation}

Within studies of collaboration, common themes are conversation analysis and hand-over/turn-taking through artifacts. Understanding the interaction between employed artifacts (e.g. technology, cards) and collaboration at a minute-tominute level are a key requirement for designing future techniques and technologies that support efficient and effective work processes that lead to valuable and usable outcomes.

Being social situations, collaborative innovation processes entail a number of social challenges, especially if groups and their members are co-located and brainstorming is done verbally, in which case participants do not have the shelter of anonymity. The most documented challenges are production blocking (only one idea can be suggested at a time; [6]), evaluation apprehension (being concerned about how others perceive one's ideas; [3]), social loafing (tendency to 
let others do the work; [18]. These challenges motivate a range of other research that investigates the role of collaboration, group size and group composition on creative output. An initial question here is often whether group-based (and thus collaborative) ideation produces more/less creative outcomes than individual ideation $[14,21]$. Whether groups can in fact achieve a higher level of creativity depends both on how creative outcomes are measured [2] and on how group members work together and is hence tied to the nature of the collaboration.

\section{Methodology: Gameful Idea Generation}

Gameful Idea Generation (GIG) is a creativity method that implements concepts from card-based innovation workshops and design games. As such, the method employs gamefulness on various levels, both for structuring the method and during the actual ideation. The method consists of three distinct phases: a Teaser, where the problem to be addressed by participants is introduced; Gamebidea, a gameful method to facilitate ideation; and Prototyping, where participants can manifest their ideas into something tangible. Both Gamebidea and the Prototyping are group-based, where about 2-3 people work together. The method of GIG is described in more detail here [19]. Gamebidea represents the phase where the actual brainstorming takes place. This phase is further broken down in three steps: Invent, Pitch and Auction. These three steps are repeated about 2-3 times depending on available time (see Fig 1).

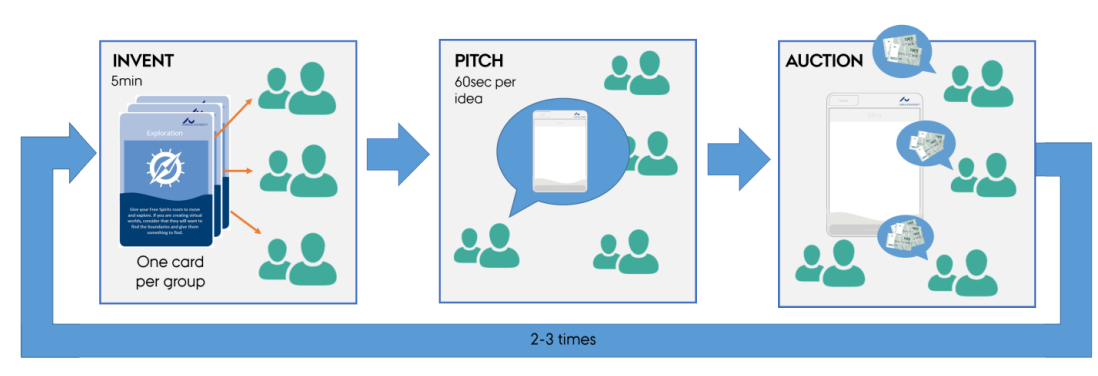

Fig. 1. Illustration of Gamebidea (based on material of the original Game of Things).

During the Invent step, individual groups have five minutes to generate as many ideas as possible addressing the given problem. Inspiration cards, which each describe one game mechanic (based on based on Marczewski's list [16]) and are randomly drawn by groups, guide this brainstorming but are also required to be incorporated in the generated ideas. Examples for such game mechanics are "Exploration", "(Experience) Points", "Unlockable Content". The latter is described as: "Add to the feeling of self expression and value, by offering unlockable or rare content for free spirits to make use of." During the ATU GIG (see next section), this mechanic was for example incorporated in an idea addressing 
plastic waste where the more non-plastic articles one buys the more discount one gets on one's purchase. After the Invent step, all groups come together for the Pitch step. Here each group presents all of their ideas in an elevator pitch format (60 seconds per idea). In the subsequent Auction step, each group can bid for individual ideas using game money (GM). This bidding and the resulting end prize determine the value (i.e. perceived quality) of an idea. The buying group gives the money to the inventing group and receives the idea which they can use in the Prototyping phase to inspire their solution. Ideas that no group bid for are discarded.

Inspired by hackathon formats, we hypothesize that including a prototyping phase in the workshop encourages grounding the ideas into something tangible and orient the idea generation towards production and presentation, rather than generating abstract ideas only. In this phase, groups have one and a half hours to build a prototype which illustrates one or multiple of their own or acquired ideas and using different analogue materials, such as paper, pens, sticky notes, cardboard, pipe cleaners, and modelling wax.

\section{Case study}

In order to explore group dynamics and behavior as well as the effect of using game mechanics as inspiration cards, we facilitated a GIG in the context of a workshop with high-school students. The event was targeted at students from the local "Academy of Talented Youth" (ATU). The problem which the students worked on was finding ways to reduce plastic waste and was introduced by showing a short video illustrating issues related to plastic waste.

Participants received no compensation for their participation (apart from lunch and refreshments provided during the event). The event lasted approximately 7 hours (including a one hour lunch break) and attracted 11 high school students (7f, $4 \mathrm{~m}$; mean $(\mathrm{M})$ age $=17.5$ ) resulting in four pairs (group A-D) and one group of three students (group E).

In total, 41 ideas were generated during three iterations of Gamebidea. The idea that was sold for the highest bid was worth 6,000 GM and was bought by group D. About a third of the ideas (35\%) sold for the minimum bid of 1,000 GM and about $13 \%$ sold for 4,000 GM.

Data collection Since GIGs are high-paced events that follow a tight schedule, we assessed stress levels and mental workload during the creative process. Accordingly, after the second and third round of Gamebidea as well as after the prototyping phase, participants filled in a questionnaire consisting of two scales, the DSSQ [17] to assess participants' stress level (based on task engagement, distress and worry) and the NASA TLX to assess mental workload.

To assess the level of collaboration that groups and individual participants engaged in during the ideation phase, namely Gamebidea, we draw on groupbased video recordings of the Invent steps and the prototyping phase. With level, 
we refer to for how long and to what extent participants worked together during ideation (e.g. turn-taking). For this activity dimension, we coded sequences where individual participants documented ideas (i.e. wrote on idea templates) or talked (i.e. speaker shifts). Turn-taking therefore describes the division of the interaction between participants, and their dominance in each. For dominance, we defined a threshold of $60 \%$, meaning in order for one participant to be considered as dominating a particular measure (e.g. talk) he or she had to be active in that way at least $60 \%$ of the total time both participants engaged in that activity. Codings of the activity dimension are neither mutually exclusive within nor across each other. This means that a participant could be both documenting an idea and talking at the same time.

\section{$4.1 \quad$ Results}

Results of our quantitative data analysis regarding intra-group dynamics during the GIG are grouped into three categories: performance (i.e. creative outcomes; quality of ideas), collaboration, and experience of individual participants.

Performance For evaluating performance in GIG, we consider game money (GM) earned per group, and number of ideas sold per group in addition to number of ideas generated.

Of the 41 ideas that were generated during the Gamebidea phase, the groups came up with 13 ideas in the first iteration (round one, R1), 18 in round two (R2), and 10 in round three (R3). Four ideas that were generated during Invent phases ended up not being presented by the respective groups in the Pitch phase, which meant that they were not offered in the Auction. Of the remaining 37, six ideas were discarded, meaning that no group bid for them (one in R1, three in $\mathrm{R} 2$ and two in R3). The average number of ideas generated was 2.7 per group per round, and 8.2 in total per round. Except for one group, all groups produced a slightly larger number of ideas in R2 (M: 3.6) than in R1 (M: 2.6). Group E can be seen as an outlier since they generated 5.3 ideas per round (R1: 6, R2: 7; R3: 3). In the third and last round, the number of generated ideas went down to the level of R1 (avg. 2.0) and for the case of group E even lower than that.

Based on the value ideas sold for, the auction mechanic can be used to rate and prioritize novel and meaningful ideas. This value surely depends on the number of groups involved as well as the time the idea entered the auction (i.e. remaining game money of individual groups to spend on ideas). Despite these factors, we argue that the value of individual ideas provides a valid indication of a group's performance. On average, during the three rounds of Gamebidea, groups earned 13,600 GM. There was a stark contrast in the value of ideas between individual rounds. Ideas sold in R3 were the most expensive selling for on average 3,000 GM. Ideas sold for considerably less money in R1 (M: 2,111 GM) and were the cheapest in R2 (M: 1,666 GM).

The group which earned the most is clearly group $\mathrm{E}$ with $28,000 \mathrm{GM}$, whereas the least was earned by group A (5,000 GM; see Table 1). The number of ideas 
sold in the three rounds is fairly consistent, with 9 ideas being auctioned off in both R1 and R2 and 6 ideas sold in R3. There is a discrepancy between the number of ideas being generated (e.g. R1: 13) and number of ideas being sold (e.g. R1: 9). The remaining ideas were either discarded (e.g. R1: 1) or never presented (e.g. R1: 3). This discrepancy is particularly high for R2, where only $50 \%$ of generated ideas were sold (three ideas were discarded). As another concrete measure for group performance, we propose the average value for how much individual groups sold their ideas in relation to how many ideas got discarded. According to this measurement group C performed best and group D showed the poorest performance.

\begin{tabular}{lcccccccccc}
\hline & \multicolumn{3}{c}{ Ideas } & \multicolumn{2}{c}{$G M$} & \multicolumn{3}{c}{ Talk } & \multicolumn{4}{c}{ Write } \\
Group & sold & discarded & earned & Performance & R1 & R2 & R3 & R1 & R2 & R3 \\
\hline A & 2 & 1 & 5,000 & 1666.67 & 4.04 & 2.83 & 2.60 & 2.45 & 5.20 & 1.90 \\
B & 6 & 0 & 13,000 & 2166.67 & 2.97 & 1.67 & 1.80 & 6.33 & 7.33 & 4.67 \\
C & 5 & 0 & 16,000 & 3200.00 & 3.95 & 2.15 & 2.75 & 1.67 & 2.92 & 2.43 \\
D & 4 & 2 & 6,000 & 1000.00 & 2.35 & 1.27 & 1.78 & 2.72 & 3.05 & 1.32 \\
E & 6 & 3 & 28,000 & 3111.11 & 2.95 & 1.97 & 2.45 & 3.30 & 10.25 & 6.43
\end{tabular}

Table 1. Overview of group's performance during the Gamebidea phase of the ATU GIG. Talk Write in min. Performance $=$ GM earned $/($ Ideas sold + Ideas discarded $)$.

Collaboration There is a clear difference in the amount of talk over the rounds. While in R1 individual groups on average talked for about 3.25 min (49\% of round duration), they talked half as much in R2 (1.97 min; 32\%). In R3, participants again spent some more time discussing ideas (2.23 min; 40\%). The difference in time spent talking between groups is not significant.

Taking a closer look at the percentage of time spent talking during idea generation provides an indication to the level of collaboration within individual groups. Since we base our evaluation on quantitative measures only but did not transcribe actual conversation, we cannot be sure that all talk was in fact taskrelated. We term brainstormings where group members spent more than $50 \%$ of the time talking conversational brainstorming, this occurred twice in R1 (group $\mathrm{A} \& \mathrm{C}$ ) and once in R3 (group A).

Whereas amount of talk clearly declined over rounds, the amount of time spent writing saw a peak in R2 (i.e. least writing activity in R3, most in R2, medium in R1). When looking at writing, one needs to keep in mind that the duration can actually be longer than the total brainstorming duration, since both participants could be writing at the same time. Since there are large differences in the time spent noting down ideas both across rounds as well as for individual groups, providing average numbers would not provide a clear picture of the situation. Overall, summing all rounds, group E spent the most time to document 
ideas (19.98 min), followed by group B (18.33 min). Group C \& D spent the least time documenting ideas $(7.02 \mathrm{~min} ; 7.09 \mathrm{~min})$. Representative for these great differences, the time spent noting down ideas in the second round increased by more than $300 \%$ for group E (R1: $3.30 \mathrm{~min}, \mathrm{R} 2: 10.28 \mathrm{~min}$ ).

Seeing that the number of generated ideas can have an impact on the time spent documenting those, we further looked at the relationship of time spent writing and number of generated ideas. Except for group A, who spent 2.45 min documenting one idea in R1, all groups on average spent about $0.64 \mathrm{~min}$ of writing per idea. R3 sees a slight increase, with 0.82 min per idea.

Regarding dominance in talk, for the majority of groups the share in talking was about equal. In six cases, one participant dominated by speaking more than $60 \%$ of the time either of them talked. Group $\mathrm{C}$ is a special case in this respect, as in all three rounds the same participant dominated the talk. The share of talk stands in contrast to the share in writing. In 10 out of 15 cases, one participant took the lead in noting down ideas on the idea templates. In five of these cases, the other participant never wrote a line. This is the case for all three rounds for group $\mathrm{C}$, where one participant (the one not dominating talk) was responsible for documenting ideas.

Individual experience As a third factor to be considered for evaluating innovation processes, we assessed participants' experience on two dimensions, stress level and mental workload. Task engagement during $\mathrm{R} 2$ was rather high (M: 18.36, standard deviation (SD): 1.36; max 20). Whereas individual participants indicated rather similar task engagements for R2, task engagement for both R3 and during prototyping $(\mathrm{P})$ varied a lot (R3 M: 15.09, SD: 4.30; P M: 14.82, SD: 4.35). This contrast also applies within groups. Difference in task engagement within groups was especially high during prototyping (A: 7, D: 10, E: 9). With the exception of two participants, task engagement slightly declined from R2 to R3 (M: 15.09). Although showing a slight overall decrease during prototyping, engagement remained considerably high.

The second stress dimension assesses distress regarding tension and participants' confidence in their (task-relevant) abilities. Across R2 (M: 10.36, SD: 3.29; max. 20), R3 (M: 10.45; SD: 2.91) and prototyping (M: 8.64; SD: 2.69) distress was mediocre, meaning that they neither felt distressed about having to perform well nor were they indifferent about their success in the given task. Distress in R2 varied quite a bit (min: 4; max: 16).

Worry, the third stress dimension, assesses to what extent people are worried about the impression they are making towards others (i.e. self-focus). Worry was rated mediocre during $\mathrm{R} 2$ (M: 11.55, SD: 3.27; max. 20), R3 (M: 9.82, SD: 2.52) and prototyping (M: 10.64, SD: 2.69) and varied the most during R2. Within groups, worry was rather similar across all steps for groups $\mathrm{B}, \mathrm{C}$ and $\mathrm{E}$, but varied quite a bit for groups A (avg. diff. 7.00) and D (avg. diff. 6.33).

To the question as to how successful they thought their groups were, individual participants responded with rather low ratings in all rounds (R2: 7.09; R3: 8.55; P: 7.94; max. 20). Group C (M: 13.5) \& D (M: 10.75) seemed mildly 
satisfied with their performance. Despite this low satisfaction regarding own performance, participants indicated low to very low frustration during R2 (M: 6.27), R3 (M: 3.27) and prototyping (M:3.73). Mental demand averaged on a moderate level with slightly decreasing over rounds (R2 M: 12.00; R3 M: 10.73; P M: 9.91). Apart from group B in R3, all groups were equally (dis-)satisfied with their performance during R2 and R3.

\section{Discussion}

Departing from previous work on differences between individual and group-based brainstorming which suggest a higher performance for individual brainstorming, it might be inferred that participants in group E worked individually. Yet from their amount of talk, it becomes clear that they did collaborate. In particular for round two and three this collaboration served more the purpose of receiving feedback on (individual) ideas instead of developing ideas together and hence had a support function. This observation applies to the majority of the groups during round two and round three.

Based on the example of group A, which came second to last according to our proposed performance measure, it can be posited that conversational brainstorming (i.e. extensively discussing ideas) does not necessarily result in a high performance during creative processes. Moreover, the group that talked the least over all rounds (group D) showed the lowest performance.

Participants in group $\mathrm{C}$ showed a clear division of work between talking and documenting. Along with the decline in talk and increase in writing is the development that previously less active participants became more active in the latter round, making participation within groups more balanced than in the first round. One explanation for this might be that after having witnessed that others welcome innovative, "funny" ideas, individual participants felt a desire to be recognized for good ideas (i.e. gain status; [5]).

Money spent and money earned directly influence each other since earned money is used to purchase more ideas in the Auction of the subsequent round. It is further determined by the strategy of the respective group; (a) ending up with as much money as possible or (b) collecting the most valuable (and thus "best") ideas. The fact that not all generated ideas were sold during the respective rounds indicates that participants actually did select ideas, hence moving on from divergent thinking to convergent thinking. Further, the varying prizes can be interpreted as a result of various degrees of interests and hence perceived value in a respective idea. The value can thus be used to prioritize and even estimate the perceived quality of the ideas. During the second round this selection was especially prevalent (e.g. three discarded ideas in R2). This selective behavior might also be due to groups wanting to save some game money for the last round.

Those groups (B \& E) that indicated on average a high task engagement during R2 and R3 performed rather well according to the purchase value of their ideas. Group A \& D, which indicated a mediocre task engagement performed 
worst, which indicates a relationship between task engagement and performance during creative processes.

Reflecting on the low satisfaction in contrast to the rather high task engagement, it might be posited that participants' critical self-evaluation motivated them to keep up their efforts. While we cannot be certain about this, we speculate that the inter-group competition contributed to this motivation. Group E showed a clear discrepancy between satisfaction with own performance (M: 1.67) and actual performance (placed 2nd). Group C, however, seemed to be both aware of and satisfied with (M: 13.5) their good performance (1st).

Our findings indicate that during gameful collaborative ideation processes, groups show various forms of collaboration (potentially oriented on their game strategy) and adapt these over time. Selection and development of ideas can be supported by gameful means, demonstrated here by exchanging (= buying) other groups' ideas. In addition to drawing on collaborative aspects, support for critical self-evaluation may push for improved quality of the work (as studied e.g. in connection with collaborative writing [20]). With support from further empirical work we hope to be able to provide a clear set of design guidelines for gameful collaborative technologies.

\section{Conclusion}

This paper has presented insights from a gameful creativity method, named Gameful Idea Generations. The analysis concentrated on intra-group collaboration and evaluation of the creative process. As part of the analysis, we proposed a method to assess the performance of a group in relation to the quality of ideas produced.

Being aware that this study was conducted in a rather special context (different from a hackathon) with a small and homogeneous user group, we plan on running more Gameful Idea Generations while varying user demographics (e.g. elderly, design students) and study designs such as including control groups in order to investigate the effect of certain game mechanics. To that end, we also

plan to explore both cross-cultural and cross-generational influences on promoting creativity through gamefulness.

\section{References}

1. Brandt, E., Messeter, J.: Facilitating Collaboration through Design Games. In: Proceedings of PDC'04. pp. 121-131. ACM (2004)

2. Chung, W.: Effective ideation method for collective creativity. In: International Conference on Applied Human Factors and Ergonomics. pp. 153-161. Springer (2018)

3. Connolly, T., Jessup, L.M., Valacich, J.S.: Effects of anonymity and evaluative tone on idea generation in computer-mediated groups. Management science 36(6), 689-703 (1990)

4. Csikszentmihalyi, M.: Flow: The psychology of optimal experience: Finding flow (1990) 
5. Dennis, A.R., Reinicke, B.A.: Beta versus HVS and the Acceptance of Electronic Brainstorming Technology. MIS Quartely 28(1), 1-20 (2004)

6. Diehl, M., Stroebe, W.: Productivity loss in brainstorming groups: Toward the solution of a riddle. Journal of personality and social psychology 53(3), 497 (1987)

7. Easterday, M.W., Gerber, E.M., Rees Lewis, D.G.: Social innovation networks: A new approach to social design education and impact. Design Issues 34(2), 64-76 (2018)

8. Gandolfi, E.: You have got a (different) friend in me: Asymmetrical roles in gaming as potential ambassadors of computational and cooperative thinking. E-Learning and Digital Media 15(3), 128-145 (2018). https://doi.org/10.1177/2042753018757757

9. Halskov, K., Dalsgård, P.: Inspiration card workshops. In: Proceedings of the 6th conference on Designing Interactive systems. pp. 2-11. ACM (2006)

10. Hornecker, E.: Creative Idea Exploration within the Structure of a Guiding Framework: The Card Brainstorming Game. In: Proceedings of TEI'10. pp. 101-108. ACM (2010)

11. Hornecker, E., Buur, J.: Getting a Grip on Tangible Interaction: A Framework on Physical Space and Social Interaction. In: Proceedings of the SIGCHI conference on Human Factors in computing systems. pp. 437-446. ACM (2006). https://doi.org/10.1145/1124772.1124838

12. Jackson, L.A., Witt, E.A., Games, A.I., Fitzgerald, H.E., Von Eye, A., Zhao, Y.: Information technology use and creativity: Findings from the Children and Technology Project. Computers in Human Behavior 28(2), 370-376 (2012). https://doi.org/10.1016/j.chb.2011.10.006

13. Kultima, A.: The Organic Nature of Game Ideation: Game Ideas Arise from Solitude and Mature by Bouncing. In: Proceedings of the international academic conference on the future of game design and technology. pp. 33-39. ACM (2010)

14. Lee, H., Parsons, D., Kwon, G., Kim, J., Petrova, K., Jeong, E., Ryu, H.: Cooperation begins: Encouraging critical thinking skills through cooperative reciprocity using a mobile learning game. Computers \& Education 97, 97-115 (2016)

15. Lucero, A., Arrasvuori, J.: PLEX Cards: A Source of Inspiration When Designing for Playfulness. In: Proceedings of the 3rd International Conference on Fun and Games. pp. 28-37. ACM (2010)

16. Marczewski, M.: New downloadable version of the gamification inspiration cards on sale, https://www.gamified.uk/2018/11/02/new-downloadable-version-of-thegamification-inspiration-cards-on-sale/

17. Matthews, G., Szalma, J.L., Panganiban, A.R., Neubauer, C., Warm, J.S.: Profiling Task Stress with the Dundee Stress State Questionnaire. Psychology of Stress pp. 49-91 (2013)

18. Paulus, P.B., Dzindolet, M.T.: Social influence processes in group brainstorming. Journal of Personality and Social Psychology 64(4), 575 (1993)

19. Thiel, S.K., Falk-Olesen, J., Halskov, K.: Gameful idea generations. In: Under Review (2019)

20. Türkay, S., Seaton, D., Ang, A.M.: Itero: A revision history analytics tool for exploring writing behavior and reflection. In: Extended Abstracts of the 2018 Conference on Human Factors in Computing Systems. pp. LBW052:1-LBW052:6. CHI EA '18, ACM, New York (April 2018). https://doi.org/10.1145/3170427.3188474

21. Voiskounsky, A.E., Yermolova, T.D., Yagolkovskiy, S.R., Khromova, V.M.: Creativity in online gaming: Individual and dyadic performance in minecraft. Psychology in Russia 10(4), 40 (2017) 
22. Williams, T.M., Fleming, J.W.: Methodological Study of the Relationship between Associative Fluency and Intelligence. Developmental Psychology 1(2), 155-162 (1969) 